\title{
THE STATE OF REPRODUCTIVE HEALTH OF WOMEN WITH HYPERPROLIFERATIVE PATHOLOGY OF THE ENDOMETRIUM
}

D0I: 10.36740/WLek202109109

\author{
Iryna M. Nikitina', Nataliia Ye. Horban², Kseniia V. Mykytyn', Kateryna 0. Kondratiuk ${ }^{3}$, Svitlana A. Smiian', \\ Nataliia V. Kalashnyk', Nadiya V. Gerevich ${ }^{2}$ \\ 'SUMY STATE UNIVERSITY, SUMY, UKRAINE \\ 2STATE INSTITUTION «INSTITUTE OF PEDIATRICS, OBSTETRICS AND GYNECOLOGY NAMED AFTER ACADEMICIAN OM LUKYANOV ACADEMY \\ OF MEDICAL SCIENCES OF UKRAINE», KYIV, UKRAINE \\ ${ }^{3}$ BOGOMOLETS NATIONAL MEDICAL UNIVERSITY, KYIV, UKRAINE
}

\begin{abstract}
The aim: To study and analyze the dynamics of women's reproductive health in Ukraine as a factor in population reproduction and to study the features of menstrual disorders in Ukraine in 2010-2019. In women of reproductive age with hyperproliferative pathology of the endometrium without atypia.

Materials and methods: A descriptive epidemiological study was conducted using data from the departmental statistical reporting of the Ministry of Health of Ukraine on the number of cases of gynecological pathology for 2010-2019. The peculiarities of menstrual function in patients of reproductive age with atypical hyperproliferative pathology of the endometrium were studied. We examined 84 patients of the gynecological department of the Sumy Regional Clinical Perinatal Center with a diagnosis of hyperproliferative pathology of the endometrium without atypia, who sought medical help during 2017-2020 for hyperplastic processes of the endometrium in reproductive age (18 to 49 years). All patients were divided into three groups, up to group I women with endometrial hyperplasia without atypia (HGE) ( $\mathrm{n}=30)$; Group II - patients diagnosed with endometrial polyps $(n=30)$; group III included patients with combined hyperproliferative pathology (uterine body polyps and endometrial hyperplasia without atypia) $(n=24)$.

Results: As a result of the conducted systematic analysis it was established: the higher the prevalence of PMC, the higher the frequency of diseases of the genitourinary system and some gynecological diseases. This is confirmed by the calculated coefficients of correlation of the prevalence of PMC with the frequency of diseases of the genitourinary system $(r=0.75, p<0.001)$, salpingitis $(r=0.63, p<0.001)$ and endometriosis $(r=0.42, p<0,05)$.

The assessment of the relative risk of gynecological diseases has shown that the greatest attention needs to be paid to improving the diagnosis of infertility and endometriosis, as well as the prevention and treatment of salpingitis and uterine cancer. When women with menstrual disorders go to the gynecologist, it is necessary to pay attention to the presence of hyperproliferative pathology, and if women have other chronic diseases, offer rehabilitation of these foci, which can further have a positive effect on reproductive health. Conclusions: The highest rates of menstrual irregularities were found in women with endometrial hyperplasia without atypia and a combination diffuse hyperplasia with endometrial polyps in contrast to patients with only endometrial polyps. Menstrual irregularities can be considered as a marker and indicator of dysfunction of the genitourinary system and the presence of a hyperproliferative process at the prehospital stage.
\end{abstract}

KEY WORDS: menstrual disorders; hyperproliferative pathology of the endometrium; endometrial polyp; hyperplastic processes of the endometrium; morphological changes of the endometrium

Wiad Lek. 2021;74(9 p.l):2076-2081

\section{INTRODUCTION}

Given the low birth rate in Ukraine, the problem of maintaining reproductive health is extremely relevant and acquires high medical and social significance, in turn, is a qualitative criterion for the reproduction of the population at the population level. In the context of economic turmoil, high mortality rates and declining birth rates, reproductive health issues are acute, which has been in the focus of international attention for some time and is being implemented by the "Action Plan on Sexual and Reproductive Health and Supporting the Implementation of the Agenda, sustainable development sector for the period up to 2030 in Europe - do not leave anyone unattended $»[1-3]$.

Indicators of reproductive health as a component of population reproduction include the incidence of women, in particular in the pre-pregnancy period, the number of abortions, infertility [4]. The specificity of a woman's reproductive health is due to the peculiarities of the realization of her reproductive function. Reproductive health forms the basis of demographic potential and is a necessary prerequisite for population growth, renewal of its quantitative and qualitative composition, which meets socio-demographic needs.

Infertility issues range from $9 \%$ to $18 \%$ in the general population and create a significant financial burden even in countries with a high level of economy [5]. An urgent medical and social problem is hyperproliferative pathology of the endometrium, which has a high share in the structure of gynecological morbidity and is accompanied by the risk of its malignant transformation, as well as the 
level of inflammatory diseases of the female genital area $[6,7]$. To date, the situation remains unstable, debatable and requires further search for solutions $[8,9]$. One of the main conditions for the birth of a healthy child is the gynecological health of the mother. That is why the aim of the work was to assess the state of gynecological health of women [10]. Unfortunately, in recent decades in Ukraine there is a trend of negative levels of reproductive health due to the negative impact of socio-economic instability in the country, falling living standards, deteriorating environmental living conditions of the population. In this regard, the problem of maintaining reproductive health as a factor in population growth, increasing the birth rate is a strategic direction that can help reduce the rate of reproductive losses [11]. That is why the aim of the work was to assess the state of gynecological health of women.

Menstrual dysfunction is one of the leading pathologies of the female genital area. Researchers in many countries around the world are paying attention search for modern diagnostic criteria, trigger factors and optimal treatment concepts. Thus, in a recent two-year US survey, it was found that among 20 million medical visits for gynecological diseases, $19.1 \%$ of cases were related to menstrual irregularities (PMC), and abnormal uterine bleeding is the cause of $25 \%$ of gynecological operations, and often radical interventions that deprive women of the ability to realize their reproductive potential, so in the UK is also indicated as the most important cause of hysterectomies [12]. About one billion dollars a year are insurance payments associated with abnormal uterine bleeding, which correlates with low socioeconomic status, psychological stress and abdominal pain $[13,14]$.

One of the most likely causes of menstrual irregularities is endometrial hyperplasia (EH). This is the most common pathology of the uterine body in women of childbearing age. According to the scientific literature, $\mathrm{EH}$ is $15-40 \%$ of all gynecological pathology $[4,15]$. Hyperproliferative pathology of the endometrium (uterine body polyps and endometrial hyperplasia) occupies a special place among the risk factors for endometrial cancer [16]. Despite numerous studies, neither the etiology nor the pathogenesis of endometrial hyperplastic processes has been elucidated to date, so treatment options are not

fully substantiated. Traditionally, the leading role in the pathogenesis of endometrial hyperplasia in patients of reproductive age is attributed to the increased estrogen concentrations arising from the absence or insufficient antestrogenic effect of progesterone. The results of scientific researches of the last years testify in favor of infectious-inflammatory concept of developing the hyperplastic processes of genitals $[3,16]$. Chronic inflammatory diseases of the genitals are the basis for the occurrence of infertility, disorders of menstrual function, hyperplastic uterine processes and endometrium [17].

\section{THE AIM}

To study and analyze the dynamics of women's reproductive health in Ukraine as a factor in population reproduction and to study the features of menstrual disorders in Ukraine in 2010-2019. In women of reproductive age and with hyperproliferative pathology of the endometrium without atypia.

\section{MATERIALS AND METHODS}

To understand the situation with PMC on the territory of Ukraine, a descriptive epidemiological study was conducted using statistical data for 2010-2019. The scientific work uses data from the departmental statistical reporting of the Ministry of Health of Ukraine on the number of cases of gynecological pathology for 2010-2019. The number of women of the appropriate age was determined according to the State Statistics Service of Ukraine. The relative risk of pathology (taking into account the $95 \%$ confidence interval) in 2010-2019 in all oblasts was assessed in comparison with the general indicators of Ukraine. Information on women with PMC was taken from the departmental statistical reporting of the Center for Medical Statistics of the Ministry of Health of Ukraine, published in the collections «Health status of women in Ukraine» for 2010-2019. The number of women of the appropriate age was determined according to the State Statistics Service of Ukraine. The fertile age in this study was limited to $18-49$ years.

Peculiarities of menstrual function in patients of reproductive age with nonatypical hyperproliferative pathology of the endometrium were also studied. We examined 84 patients of the gynecological department of the Sumy Regional Clinical Perinatal Center with a diagnosis of hyperproliferative pathology of the endometrium without atypia, who sought medical care during 2017-2019 for hyperplastic processes of the endometrium in reproductive age. The age of women ranged from 18 to 49 years (average $34.52 \pm 2.51$ ). All procedures were carried out in accordance with the ethical standards of the responsible committee on human experimentation and with the Helsinki Declaration of 1975, as revised in 2000.

All patients were divided into three groups, up to group I women with endometrial hyperplasia without atypia (HGE) $(\mathrm{n}=30)$; Group II - patients diagnosed with endometrial polyps $(\mathrm{n}=30)$; group III included patients with combined hyperproliferative pathology (uterine body polyps and endometrial hyperplasia without atypia) $(\mathrm{n}=24)$.

The criteria for inclusion of patients in the experiment was histologically confirmed the diagnosis of endometrial hyperplastic process (with simple and complex endometrial hyperplasia without atypia according to the classification of WHO endometrial pathology (2014)) [17].

Exclusion criteria were of women with alimentary-constitutional obesity (BMI> 30.0), the presence of severe somatic or mental pathology.

Hysteroscopy was performed under intravenous anesthesia on hard fiber optics company "KARLSTORZ" from the 6th to the 11th day of the menstrual cycle (with a preserved menstrual cycle). After complete removal of pathologically altered endometrial tissue, its histological examination was performed. The material was fixed in a buffered $10 \%$ formalin solution. 
Pipelle biopsy was performed on days 19-23 menstrual cycle using an aspiration probe. The aspiration probe was inserted into the uterine cavity in an assembled form to the bottom area and the contents were aspirated with a piston, resulting in the effect of "suction" to the walls of the uterine cavity and through the existing perforation material which enters the probe. After performing 2-3 aspirations, the conductor was removed and the probe was removed from the uterine cavity. The resulting material is placed in a vial with a $10 \%$ solution of neutral formalin.

Histological examination of endometrial tissue was performed according to the generally accepted method [18] on the basis of the Department of Morphology of the Sumy State University.

The surgical material and endometrial tissue obtained by hysteroscopy and aspiration biopsy of the endometrium were subjected to morphological examination. Biopsy material was fixed in $10 \%$ neutral formalin. Further processing was performed according to the standard generally accepted unified method [18]. Examination of histological specimens was performed using a Nikon Eclipse light microscope (CiE) using a digital video camera according to the software manufacturer's recommendations.

For electron microscopic examinations, pieces of endometrium were removed, fixed in $2.5 \%$ glutaraldehyde solution, and postfixed with $1 \%$ osmium tetraoxide solution on phosphate buffer. Further processing was performed according to the generally accepted method [19]. Ultrathin sections made on an ultramicrotome UMPT-7 were contrasted with uranyl acetate, lead citrate according to the Reynolds method and studied under an electron microscope PEM-125K.

For detailed analysis and photo documentation, the image from the microscope was transferred to a computer monitor using a VISION Color CCD Camera and InterVideoWinDUR.

All diagnostic and therapeutic manipulations were performed after the written consent of the patients for examination and treatment in accordance with the protocol approved by the Bioethics Committee (№53 / 03.06.2019). Consent to surgical treatment was obtained before treatment.

The obtained data were processed using standard statistical methods using a personal computer HP PREMIER EXPERIENCE with MicrosoftWord 2010, the graphics were built using Microsoft Excel. Statistical processing was performed by the program Statistics 10 .

\section{RESULTS}

The frequency of PMC for 2010-2019 was $15.96 \pm 0.01$ among 1000 women of childbearing age (Fig. 1). In terms of regions, ten-fold fluctuations of the indicator values were observed - from the smallest in Luhansk region (5.90 \pm 0.05$) \%$ to the largest in Dnipropetrovsk region $(26.16 \pm 0.06) \%$ and in Kyiv $(29.84 \pm 0,07))$. The difference between the smallest and largest value of the indicator was threefold.

The ratio of risks of $\mathrm{PMC}$ among patients of childbearing age for the period 2010-2013 in most regions was lower than the general figure for Ukraine. The lowest indicator of relative risk was in Ternopil region (OR - 0.56 [95\% CI 0.54-0.57], $\mathrm{p}<0.05$ ), Poltava (OR - 0.56 [95\% CI 0.55-0,57], $\mathrm{p}<0.05)$, Sumy (OR -0.57 [95\% CI 0.57-0.58], $\mathrm{p}<0.05$ ), Rivne (OR - 0.61 [95\% CI 0,59-0.62], $\mathrm{p}<0.05$ ). At the same time on the territory of other regions (Vinnytsia (OR - 1.05 [95\% CI 1.04-1.07], p<0.05), Kharkiv (OR 1.12 [95\% CI $1.11-1.13$ ], $\mathrm{p}<0.05)$, Lviv (OR - 1.14] (95\% SI 1.13-1.15], $\mathrm{p}<0.05$ ), Cherkasy (OR - 1.26 [95\%) CI $1.24-1.28$ ], $\mathrm{p}<0.05$ ), Dnipro (OR 1.49 [95\% CI 1.48-1.50], $\mathrm{p}<0.05$ ), Zhytomyr (OR 1.56 [ 95\% CI 1.54-1.5], $\mathrm{p}<0.05$ ).

The ratio of risks of PMC among women of childbearing age for the period 2014-2019 repeats the figures for consecutive 4 years, and in Kyiv it was (OR - 1.93 [95\% CI 1.91-1.94], $\mathrm{p}<0,05)$. The ratio of risks of PMC among women of childbearing age for the period 2014-2019 compared to 2010-2013 in Ukraine as a whole (OR - 1.07 [95\% CI 1.06-1.07], p<0.05) and in Kyiv (OR 1.00 [95\% CI $0.99-1.01], \mathrm{p}<0.05$ ), as in most regions it was increased. The worst indicators of the ratio of PMC risks were observed in Dnipro (OR - 1.35 [95\% CI 1.34-1.37], p<0.05), Poltava (OR - 1.31 [95\% CI 1.28-1.34], $\mathrm{p}<0.05$ ), Ivano-Frankivsk (OR - 1.20] (95\% CI 1.17-1.23], p<0.05), Khmelnytsky (OR - 1.19 [95\%) CI 1.16-1.21], p<0.05), Mykolayiv (OR 1.16] (95\% CI 1.13-1.19], $\mathrm{p}<0.05)$ and Sumy (OR - 1.14 [95\% CI 1.12-1.17], $\mathrm{p}<0.05)$ areas. The decrease in OR in 2014-2019 compared to 2010-2013 was recorded in (OR - 0.88 [95\% CI 0.86-0.89], $\mathrm{p}<0.05$ ), Transcarpathian (OR - 0,89 [95\% CI 0.87-0.91], $\mathrm{p}<0.05$ ), Chernivtsi (OR 0.93 [95\% CI 0.91-0.95], p<0.05), Kropyvnytskyi (OR 0.94 [95\% CI 0.92-0.97], $\mathrm{p}<0.05$ ) areas. In Kyiv and Lviv region, the prevalence of PMC in women did not change, remained at a high level.

The next stage of scientific research is to study the features of menstrual function in patients of reproductive age with hyperproliferative pathology of the endometrium without atypia. In a detailed analysis of the division of PMC by its forms, it was shown that the most frequently observed irregular cycle and menorrhagia $(11.96 \pm 2.39)$ and $(11.41 \pm 2.34) \%$, respectively, among women from all three study groups). Chronic inflammatory diseases of the pelvic organs were most often determined in the subjects (salpingitis, $21.6 \%$ ). In subjects who had menstrual irregularities, hyperproliferative processes of the endometrium were more often registered $(10.8 \%$ vs. $3.1 \%, \mathrm{p}<0.01)$, and in those who had an inflammatory process of the pelvic organs, cervical dysplasia was more common ( $19.8 \%$ vs. $4.7 \%, \mathrm{p}<0.001$ ), chronic diseases of the genitourinary system $(7.1 \%$ vs. $3.2 \%$, $\mathrm{p}<0.05)$ and digestive organs $(17.3 \%$ vs. $9.6 \%, \mathrm{p}<0,01)$, which indicates the pathogenetic role of inflammatory factor in the development of gynecological pathology.

At the same time, when considering certain groups, there was a tendency to increase the share of women with irregular cycles in the group of HPE compared with the group of women with uterine polyps ( 18.3 vs. $8.1 \%, p=0.16)$ and to increase the share of women with menorrhagia in group of women with combined HPE in contrast to the group with polyps of the uterine body ( 17.7 vs. $6.5 \%, \mathrm{p}=0.10)$ (Fig. 2$)$. 

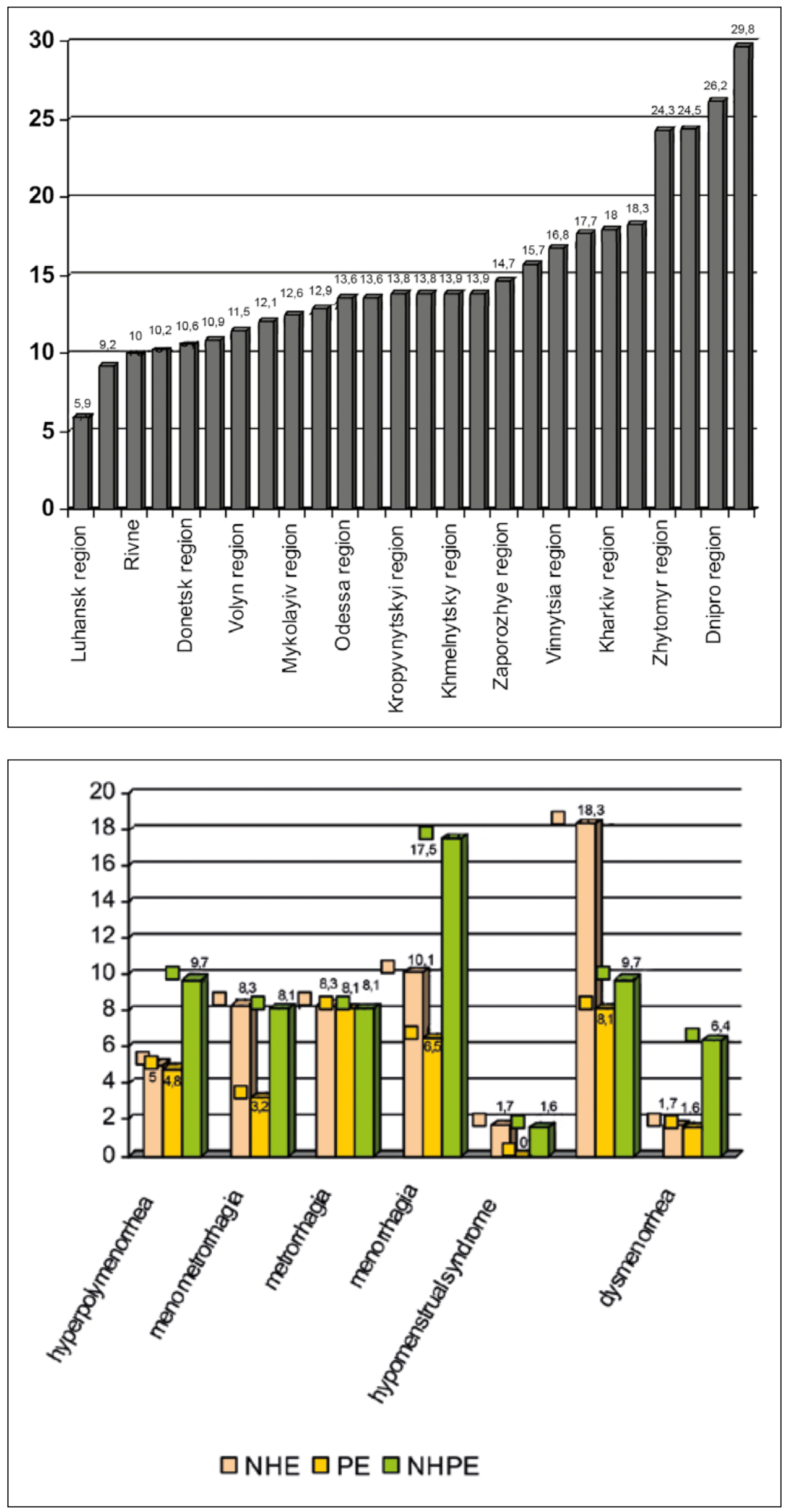

Fig. 1. Indicator of the frequency of PMC in women of childbearing age in the regions of Ukraine (per 1,000 women aged 18-49) during 2010-2019

Fig. 2. Menstrual irregularities in women of the examined groups 
In other cases, no statistically significant difference was observed. If we summarize all the PMC, we can see a clear difference between the groups of women surveyed. Thus, the highest proportion of PMC was observed in women with combined HPE $(61.3 \pm 6.2) \%$ and in patients with morphological signs of HPE $(53.3 \pm 6.4) \%$, while in patients with of which uterine body polyps were verified, this diagnosis was established only in one third $(32.3 \pm 5.9) \%, p=0.002$ and $p=0.03$, respectively). This can be considered as one of the important clinical markers of the presence of hyperplastic process of the endometrium, even in the prehospital stage and in the absence of technical capabilities for additional diagnostic methods.

\section{DISCUSSION}

As a result of the conducted systematic analysis it was established: the higher the prevalence of PMC, the higher the frequency of diseases of the genitourinary system and some gynecological diseases. This is confirmed by the calculated coefficients of correlation of the prevalence of $\mathrm{PMC}$ with the frequency of diseases of the genitourinary system $(r=0.75, p<0.001)$, salpingitis $(r=0.63, p<0.001)$ and endometriosis $(\mathrm{r}=0.42, \mathrm{p}<0,05)$.

Summarizing the results of calculating the risk ratio of PMC with similar calculations of the risk ratio of salpingitis, endometriosis and diseases of the genitourinary system among the regions of Ukraine in nine observed a reduced risk ratio of all the above pathological conditions (including Sumy, Chernihiv and Poltava regions, five western oblasts Volyn, Zakarpattia, Rivne, Ternopil, Khmelnytsky, and Luhansk regions) and in four (Dnipro region, Zhytomyr region, Kharkiv region, Cherkasy region and Kyiv) - increased, which requires the application of urgent measures by health care organizers in within the framework of the application of screening programs for the identification of risk groups for the occurrence of this pathology, as well as the use of treatment and prevention measures to improve reproductive potential.

The assessment of the relative risk of gynecological diseases has shown that the greatest attention needs to be paid to improving the diagnosis of infertility and endometriosis, as well as the prevention and treatment of salpingitis and uterine cancer. When women with menstrual disorders go to the gynecologist, it is necessary to pay attention to the presence of hyperproliferative pathology, and if women have other chronic diseases, offer rehabilitation of these foci, which can further have a positive effect on reproductive health.

\section{CONCLUSIONS}

1. It is established that the frequency of PMC among women of reproductive age in Ukraine in 2010-2019 was $(15.96 \pm 0.01) \%$.

2. PMC can be considered as an important marker and indicator of trouble in the functioning of the entire genitourinary system in the prehospital stage, which is correlated between the prevalence of PMC and the increasing incidence of diseases of the genitourinary system $(\mathrm{r}=0.75, \mathrm{p}<0.001)$, salpingitis $(\mathrm{r}=0.63$, $\mathrm{p}<0.001)$ and endometriosis $(\mathrm{r}=0.42, \mathrm{p}<0.05)$.

3. $\mathrm{PMC}$ was determined in all groups of women with hyperproliferative pathology of the endometrium without atypia, with the highest rates in women with combined HPE $(61.3 \pm 6.2) \%$ and in patients with endometrial hyperplasia without atypia $(53.3 \pm 6.4) \%$ in contrast to patients with polyps of the uterine body $(32.3 \pm 5.9) \%$, $\mathrm{p}=0.002$ and $\mathrm{p}=0.03$, respectively).

4. Menstrual irregularities can be considered as a marker and indicator of dysfunction of the genitourinary system and the presence of a hyperproliferative process in the prehospital stage.

\section{REFERENCES}

1. Akrish S., Eskander-Hashoul L., Rachmiel A. et al. Clinicopathologic analysis of verrucous hyperplasia, verrucous carcinoma and squamous cell carcinoma as part of the clinicopathologic spectrum of oral proliferative verrucous leukoplakia: A literature review and analysis. Pathol. Res. Pract., 2019; 215 (12):152670. doi:10.1016/j.prp.2019.152670.

2. Farhane F.Z., AlamiZ., Bouhafa T. et al. Primary squamous cell carcinoma of endometrium: case report and literature review. Pan Afr. Med. J., 2018; 4 (30): 8. doi:10.11604/pamj.2018.30.8.8983.

3. Upadhyaya J.D., Fitzpatrick S.G., Islam M.N. et al. A retrospective 20year analysis of proliferative verrucous leukoplakia and its progression to malignancy and association with high-risk human papillomavirus. Head. Neck. Pathol., 2018; 12 (4): 500-510. doi:10.1007/s12105-0180893-7.

4. Cicinelli E., Matteo M., Trojano G. et al. Chronic endometritis in patients with unexplained infertility: Prevalence and effects of antibiotic treatment on spontaneous conception. Reprod. Immunol. 2018; 79 (1): e12782. doi: 10.1111/aji.12782.

5. Vovk I.B., Horban N.Y., Lysiana T.A. et al. The role of the inflammatory factor in the genesis of hyperplastic changes of endometrium in women of reproductive age. Actual Prob. Pediatr., Obstet. Gynecol. 2019; (1): 73-80. doi:10.11603/24116-4944.2019.1.10180.

6. Gorban N.Y., Vovk I.B., Hoida N.H. et al. Menstrual dysfunction: the situation in the world and in ukraine - in women of reproductive age and with atypical hyperproliferative pathology of the endometrium. Bull. Soc. Hygiene Health Protect. Organiz. Ukraine. 2019; (3): 77-83. doi: 10.11603/1681-2786.2019.3.10596.

7. Dobrokhotova Yu.E., Yakubova K.K. Microbiota of the reproductive tract and hyperplastic processes of the endometrium (literature review). RMJ "Medical Review", Gynecology. 2018; 10: 14-16.

8. Gorban N.Y., Zadorozhna T.D., Vovk I.B. et al. Morphological features of uterine polyps in females of reproductive age. Bull. Sci. Res. 2019; (2): 47-52. doi: $10.11603 / 2415-8798.2019 .2 .10267$

9. Chornenka G.M., Logach M.V. Morphological features of endometrium in women of reproductive age: analysis of morphometric indicators. Achievements of Clinical and Experimental Medicine. 2018; (3): 146-150. doi: 10.11603/1811-2471.2018.v0.i3.9275.

10. Gordts S., Grimbizis G.F., Campo R. Symptoms and classification of uterine adenomyosis, including the place of hysteroscopy in diagnosis. Fertil. Steril. 2018; 109 (3): 380-388. doi: 10.1016/j.fertnstert.2018.01.006.

11. Thompson A.B., Flowers L.C. Human papillomavirus (HPV). In: Hussen S. (eds). Sexually transmitted infections in adolescence and young adulthood. Springer, Cham. 2020: 279-297. doi: 10.1007/978-3-03020491-4_18. 
12. Wu Q., Chu Z., Han H. Primary squamous cell carcinoma of the endometrium in a woman of reproductive age: a rare case report. J. Int. Med. Res. 2018; 46 (8): 3417-3421. doi:10.1177/0300060518776579.

13. Rintala M., Vahlberg T., Salo T. et al. Proliferative verrucous leukoplakia and its tumor markers: Systematic review and meta-analysis. Head Neck. 2019: 41 (5): 1499-1507. doi:10.1002/hed.25569.

14. Tetikkurt S., Çelik E., Taş H. et al. Coexistence of adenomyosis, adenocarcinoma, endometrial and myometrial lesions in resected uterine specimens. Mol. Clin. Oncol., 2018; 9 (2):231-237. doi:10.3892/ mco.2018.1660.

15. Kurman R.J., Carcangiu M.L., Herrington C.S. World Health Organisation. Classification of tumours of the female reproductive organs. 4th Revised ed. International Agency for Research on Cancer, 2014.

16. Nikitina I., Boiko V., Smiian S. et al. Estimation to effigiency of the multifunction metod of endometriosis ovaries treatment. Wiad. Lek. 2020; 5:868-873.

17. Gorban N., Vovk I., Nikitina I. et al. Immunoglobulin indicators to viruses cytomegal and genital herpes in the blood serum of women with nonatiptcal endometrial hyperproliferative patology. Wiad. Lek. 2020; 8: 1600-1605.

18. Boichuk A.V., Shadrina V.S., Vereshchahina T.V. Hyperplasia of endometrium - a modern system-pathogenetic view on the problem (literature review): (literature review). Actual problems of pediatrics, obstetrics and gynecology, 2019; (1): 67-72. doi: 10.11603/241164944.2019.1.9906.

19. Kulavsky V.A., Pushkarev B.A., Kulavsky E.V. et al. Pathology of the endometrium: (diagnostic and therapeutic aspects). Ufa: Healthcare publishing house of Bashkortostan, 2018, 328.

The work is carried out within the framework of the research work "Optimization of diagnosis and prevention of diseases of the reproductive system and development of pathogenically grounded methods for their correction" (state registration number 011U001801).

\section{ORCID and contributionship:}

Iryna M. Nikitina: 0000-0001-6595-2502 ${ }^{A, E, F}$

Nataliia Ye. Horban: 0000-0002-3201-498X ${ }^{C, B, D}$

Kseniia V. Mykytyn: 0000-0001-9620-550X ${ }^{C, D, F}$

Kateryna O. Kondratiuk: 0000-0001-5915-1821 C,E

Svitlana A. Smiian: 0000-0002-7679-2302 ${ }^{D}$

Natalia V. Kalashnyk: 0000-0003-1515-9105 ${ }^{\mathrm{E}}$

Nadiya V. Gerevich: 0000-0002-5984-3307 ${ }^{E}$

\section{Conflict of interest:}

The Authors declare no conflict of interest.

\section{CORRESPONDING AUTHOR Iryna M. Nikitina \\ Sumy State University \\ Rymskoho-Korsakova 12, 40030 Sumy, Ukraine \\ tel: +380662947360 \\ e-mail: nikitina1med@gmail.com}

Received: 19.05 .2021

Accepted: 25.08 .2021

A - Work concept and design, B - Data collection and analysis, C - Responsibility for statistical analysis, D-Writing the article, $\mathbf{E}$-Critical review, $\mathbf{F}$ - Final approval of the article 